\title{
The Establishment of BALB/c Mouse Model Infected by Salmonella Typhimurium CVCC541
}

\author{
Xueqin Zhao, Hanna Fotina, Lei Wang, Jianhe Hu
}

\begin{abstract}
The infection of Salmonella is the main cause for gastrointestinal diseases worldwide. There are about 20 to 40 million cases with gastroenteritis caused by Salmonella infection across the world every year. In our study, we set up the Chinese Salmonella typhimurium CVCC541 infected mouse model by intraperitoneal injection with different amount of bacteria respectively. The results suggested the severity of clinical symptoms in mice was positively correlated with the dosage and duration of infection. Mice infected by $S$. typhimurium CVCC541 behaved loose coat and hunched back, sluggish, anorexic, and tendency to gather together. The necropsy results showed the severity of pathological changes was also positively correlated with the dosage of infection. The major lesion occurred in the intestine. Severe hemorrhage in the intestinal tract, especially in jejunum and ileum. Meanwhile, we found the amount of neutrophils increased, and the amounts of macrophages and $T$ lymphocytes decreased significantly in the spleen of mice infected with S. typhimurium CVCC541. We first reported the clinical symptoms and pathological damages of mice caused by the infection of S. typhimurium CVCC541 at various concentrations in detail, which would provide the reference for studies of the molecular mechanism of Salmonella infection and drug resistance.
\end{abstract}

Index Terms - Salmonella typhimurium, mouse model, pathological damage, intraperitoneal injection.

\section{INTRODUCTION}

Salmonella is a gram-negative bacterium, and it is one of the major pathogens to cause gastrointestinal diseases worldwide [1], [2]. Every year, there are about 20 to 40 million cases of gastroenteritis caused by the infection of Salmonella, and the associated economic loss is more than 2 billion US dollars, which brings serious threat to the world public health [3]. There are two Salmonella serotypes, typhimurium and paratyphoid [4], [5]. In clinic, the major serotype is typhimurium, which can due to the ingestion of contaminated food, insanitary condition of environment, antibiotic abuse etc [6], [7]. The epidemic and infection of S. typhimurium have caused huge losses to the world economic development. Recently, it has become the hot spot of public concern and scientific study. The establishment of mouse model infected

Xueqin Zhao Faculty of Veterinary Medicine, Sumy National Agrarian University, Sumy, Ukraine; College of Animal Science and Veterinary Medicine, Henan Institute of Science and Technology, Xinxiang, P. R. China Hanna Fotina Faculty of Veterinary Medicine, Sumy National Agrarian University, Sumy, Ukraine

Lei Wang College of Animal Science and Veterinary Medicine, Henan Institute of Science and Technology, Xinxiang, P. R. China

Jianhe Hu College of Animal Science and Veterinary Medicine, Henan Institute of Science and Technology, Xinxiang, P. R. China ) by S. typhimurium would be an effective approach to study the pathogenesis and drug resistance of this bacterium [8]. S. typhimurium causes self-limiting gastroenteritis in human. The mice have the similar symptoms as human after infected by $S$. typhimurium. Therefore, the study of mice infected by $S$. typhimurium may provide therapeutic strategies to the treatment of human infected by $S$. typhimurium [9]. Moreover, the mouse model could be applied for the new drug development and the improvement of treatment efficacy. BALB/c mouse is one of ideal mouse strain to study the pathogenesis of diseases. The mutation and deletion of Nramp1 gene cause mice to be susceptible to many diseases [10]. Preeti Kalia et al. reported that BALB/c mice infected by $S$. typhimurium MTCC 98 manifested high temperature, loose coat, sleepiness, and other typical typhoid symptoms; therefore, this mouse model was used for the study of the efficacy of combined treatment of Propolis and Cefixime [11].

In our study, the BALB/c mice were used. The mouse infection model was established through intraperitoneally injecting $S$. typhimurium CVCC541 at various concentrations. The pathogenesis and pathological damages to the mice were assessed in detail. The results of our study could provide information for the establishment of mouse models infected by other bacteria, and provide the reference to further study the infection of Salmonella and molecular mechanism of drug resistance.

\section{MATERIALS AND METHODS}

\section{A. Laboratory animals}

Female BALB/c mice (6 to 8 weeks old, body weight of 18 to $20 \mathrm{~g}$ ) were purchased from the Animal Center of Zhengzhou University (NO. 41003100003648). All animal studies were conducted according to the experimental practices and standards of the Animal Welfare and Research Ethics Committee at Zhengzhou University. The study was approved by the Animal Centre of Zhengzhou University.

\section{B. Bacterial culture}

S. typhimurium (CVCC541, purchased from China Veterinary Drug Control Agency) were cultured in LB medium at $37{ }^{\circ} \mathrm{C}$. When bacteria were growing in the exponential stage, they were collected after centrifuged at $8000 \mathrm{rpm} / \mathrm{min}$ [12]. The bacteria solution was diluted to $1 \times 10^{7} \mathrm{CFU} / \mathrm{mL}, 1 \times 10^{6} \mathrm{CFU} / \mathrm{mL}, 1 \times 10^{5} \mathrm{CFU} / \mathrm{mL}$, and $1 \times 10^{4}$ $\mathrm{CFU} / \mathrm{mL}$ respectively with PBS for the further mice infection experiment. 


\section{The establishment of mouse model}

Intraperitoneal injection was used for the infection of mice. Mice were divided into 5 groups randomly, 10 mice in each group. The infection dosages were $1 \times 10^{7} \mathrm{CFU} /$ mouse, $1 \times 10^{6}$ CFU/mouse, $1 \times 10^{5}$ CFU/mouse, $1 \times 10^{4}$ CFU/mouse, respectively. The mice in the control group were injected with the same volume of PBS [13].

D. The observation of clinical symptoms and survival rate

The criteria of symptoms score was set up, and the clinical symptoms of mice infected by bacteria were graded. The score criteria were sluggishness (1 point), anorexia (1 point), loose coat (1 point), rapid heart rate (1 point), tendency to gather together (1 point), hunched back (1 point) and etc. Scores could be superposed. The severer the symptoms, the higher the scores. The time of death and the number of mice were recorded as well [14].

\section{E. The organ colony colonization}

The liver, spleen and lung from infected mice were removed and grinded under sterilized condition. The equal proportion volume of PBS to the organ weight was added to the grinded tissues. The dilution gradients were $10^{-1}, 10^{-2}, 10^{-3}, 10^{-4}$ and $10^{-5}$. The $200 \mu \mathrm{L}$ organ solution was used for the cell counting.

\section{F. The observation of organ lesions}

The necropsy of dead mice was performed, and the pathological changes of mice organs were observed. The mice organs were fixed with $4 \%$ paraformaldehyde, embedded in paraffin, cut to the slices. HE staining was performed, and the microscopic pathological changes were observed [15], [16].

\section{$\mathrm{G}$. The determination of lymphocytes ratios}

The ratio of spleen lymphocytes from mice one week after infection was determined by flow cytometry. After spleen is grinded, spleen lymphocytes were filtered through 200 mesh nylon net. The cell suspension was collected, red cell lysing reagents were added and mixed thoroughly with suspension. Lymphocytes were washed and collected [17]. The antibodies of FITC-anti-Ly6G, FITC-anti-CD3, and FITC-anti-F4/80 were incubated with lymphocytes for $45 \mathrm{~min}$ at room temperature away from light. After the incubation, lymphocytes suspension was centrifuged for $5 \mathrm{~min}$ at 1000 $\mathrm{rpm} / \mathrm{min}$. the supernatant was discarded, and the pellet was washed twice with PBS. Finally, 500 $\mathrm{L}$ PBS was added into the lymphocytes pellets, and samples were examined with flow cytometer within $1 \mathrm{~h} \mathrm{[18].}$

\section{RESULT}

A. Clinical symptoms and survival rate of mice infected with S. typhimurium CVCC541

After mice were infected with $S$. typhimurium CVCC541, a variety of symptoms were observed. Mice injected intraperitoneally with bacteria at the concentration of $1 \times 10^{7}$ CFU/mouse S. typhimurium CVCC541 manifested the most severe symptoms. On the first day after injection, the mice have already manifested symptoms such as anorexia, rapid heart rate, body trembling, loose coat, and hunched back. On the fourth day, symptoms of mice were the most severe, and all mice died (Fig. 1a). Mice infected by bacteria at the concentration of $1 \times 10^{6} \mathrm{CFU} /$ mouse $S$. typhimurium CVCC541 manifested the clinical symptoms such as anorexia, and symptoms became worse over time. On the seventh day, the mortality rate of mice was $100 \%$, the minimum lethal dose of CVCC541 to mice was $1 \times 10^{6}$ $\mathrm{CFU} / \mathrm{mouse}$. Mice infected with bacteria at the concentration of $1 \times 10^{5} \mathrm{CFU} / \mathrm{mouse}$ S. typhimurium CVCC541 manifested moderate symptoms such as anorexia, tendency to gather together. On the fifth day, symptoms started to improve, and the survival rate of mice was $80 \%$ by day 7 . Meanwhile, mice infected with bacteria with the lowest concentration of $1 \times 10^{4}$ CFU/mouse $S$. typhimurium CVCC541 had mildest symptoms and there was no significant difference on the score of clinical symptoms, compared with the mice in the control group. Moreover, the survival rate of mice in this group was $100 \%$ (Fig. 1b). Mice in the negative control group did not show any adverse reaction.

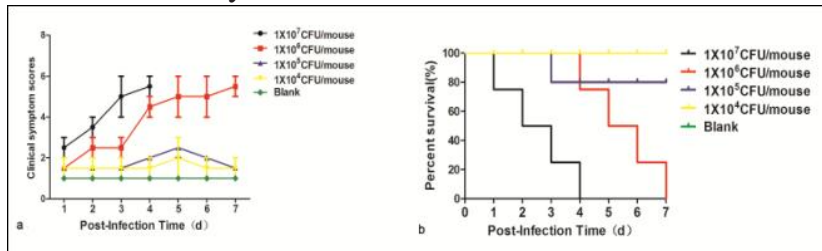

Fig.1: Clinical symptoms and survival rate of mice infected with S. typhimurium CVCC541.

(a) Clinical symptoms of mice infected with S. typhimurium CVCC541 at various concentrations;

(b) The survival difference of mice infected with $\mathrm{S}$. typhimurium CVCC541 at various concentrations.

B. The weight, bacteria content and pathological changes in mice organ

Mice infected by bacteria at the concentration of $1 \times 10^{7}$ CFU/mouse $S$. typhimurium CVCC541 manifested very severe pulmonary congestion and hemorrhage. The liver in the mice infected by bacteria at the concentration of $1 \times 10^{7} \mathrm{CFU} /$ mouse was congested with dark red color without swelling. There was no obvious pathological and weight changes in different groups ( $p>0.05$, Fig. $2 a$ and $3 c)$. The spleen in the mice infected by bacteria at the concentration of $1 \times 10^{6} \mathrm{CFU} /$ mouse was swelling significantly by eyeballing, and the proportion and weight were increased by approximately one fold ( $\mathrm{p}<0.05$, Fig. $2 \mathrm{~b}$ ). The mice spleen in other groups did not show obvious pathological changes (Fig. 3d). The accompanying pathological changes were pulmonary swelling and obviously increased weight of the lung ( $p<0.05$, Fig. $3 c$ ). Pathological changes in the lung of mice infected by bacteria at the concentration of $1 \times 10^{6}$ $\mathrm{CFU} /$ mouse and less were much milder, which indicated that low dose of $S$. typhimurium CVCC541 caused less pathological damages to the lung (Fig. 3b). The results on the amounts of colony colonized in organs of mice indicated that there were more bacteria colonized in spleen, and there was close correlation between the bacteria colony number and bacteria number infected (Fig. 2d). 
The results from the necropsy indicated that the intestine in mice infected by the bacteria at the high concentration of $1 \times 10^{7} \mathrm{CFU} / \mathrm{mouse}$ S. typhimurium CVCC541 was congested, hemorrhagic, becoming thinning, and easy to rupture. The jejunums of dead mice at $48 \mathrm{hr}, 60 \mathrm{hr}$, and $96 \mathrm{hr}$ after infection manifested very severe congestion (Fig. 3a). The results from the necropsy showed that the mice infected by the bacteria in the moderate concentration of $1 \times 10^{6}$ CFU/mouse $S$. typhimurium CVCC541 had congestion in the intestinal tract, thinner bowel wall, and full yellow sticky fluid in the intestinal cavity. The time point when mice had most severe pathological changes was at the $120^{\text {th }} \mathrm{h}$ after infection. After that time point, the pathological changes of intestine improved (Fig. 3a). The necropsy results showed that the mice infected by the bacteria at the low concentration of $1 \times 10^{5} \mathrm{CFU} / m o u s e \mathrm{~S}$. typhimurium CVCC541 did not manifest any obvious pathological changes within 6 days after infection. From the $7^{\text {th }}$ day, the intestine was congested and there were severe effusion in the peritoneal cavity. There was no pathological change found in the cecum and rectum. Intestinal pathological changes in mice infected by the bacteria at the very low concentration of $1 \times 10^{4} \mathrm{CFU} /$ mouse $S$. typhimurium CVCC541 were very mild (Fig. 3a). The intestine of mice in the negative control group was normal, no pathological changes were detected, and the bowel walls were thick and elastic (Fig. 3a).
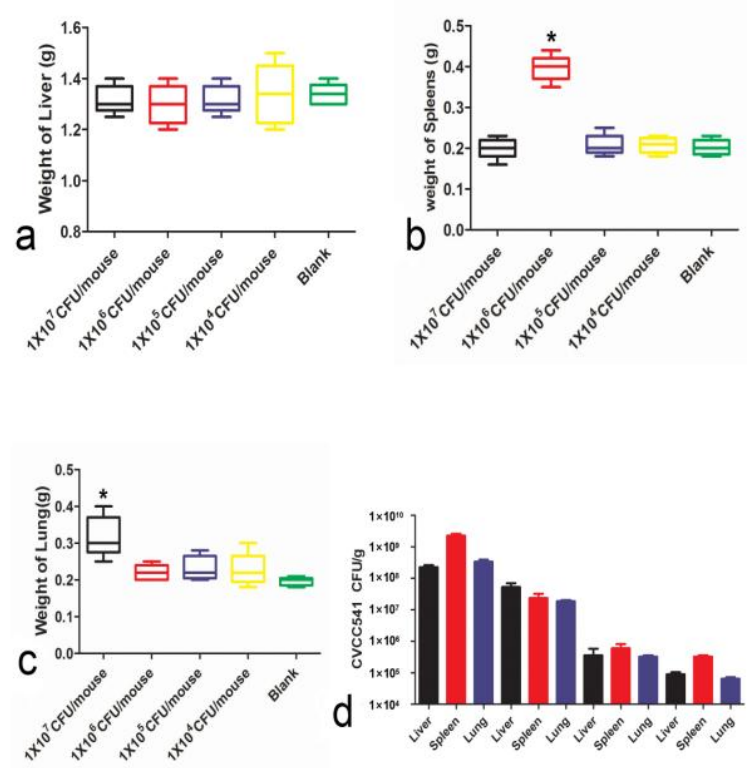

Fig. 2: The organ weights and bacteria content in mice infected with S. typhimurium CVCC541.

(a-c) The weight of liver, spleen and lung in mice infected with S. typhimurium CVCC541;

(d) The bacteria content in the liver, spleen and lung in mice infected with S. typhimurium CVCC541.

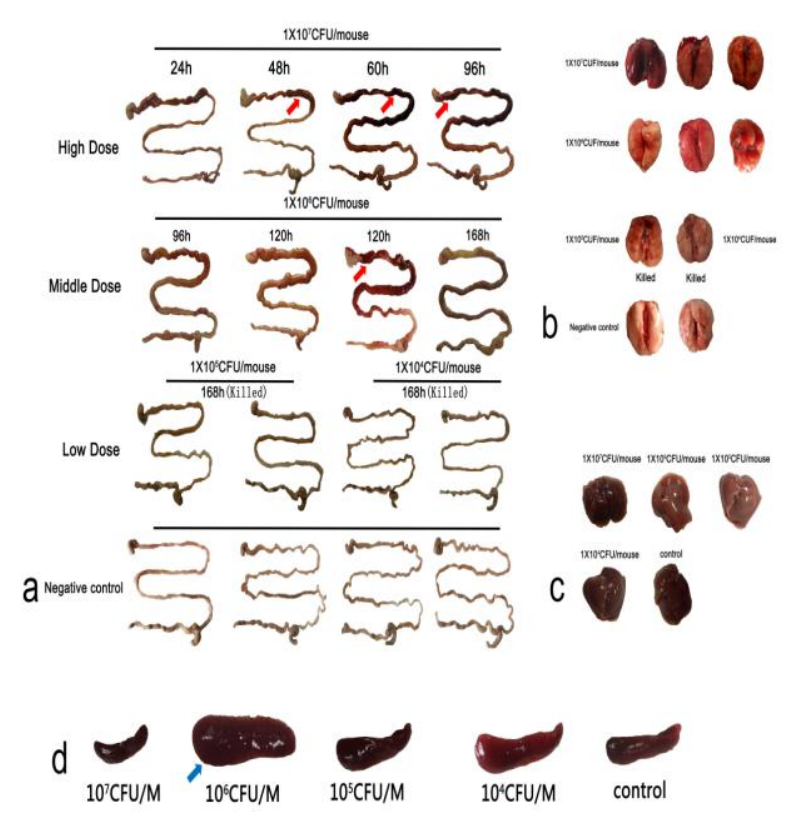

Fig. 3: Pathological changes of organs in mice infected with S. typhimurium CVCC541.

(a-d) Pathological changes of the digestive tract, lung, liver and spleen in mice.

\section{CVCC541 could cause necrotic enteritis in mice}

The pathological observation of intestines in mice infected by bacteria at various concentrations showed the absence of villi as well as the rupture of villi into the intestinal cavity, and Catarrhal enteritis have arisen in the jejunum of the mice infected by bacteria at the concentration of $1 \times 10^{7} \mathrm{CFU} /$ mouse S. typhimurium CVCC541. The epithelial cells in the jejunum were degenerated, necrotic, dropped. The lamina propria of the jejunum was congested and infiltrated by a great amount of neutrophils. The vessels were dilated and congested, and cellulose exuded in a mass. The cellulose exuded and necrotic mass condensed together to form a solid film, which was difficult to be peeled off. All these were the typical pathological changes of necrotic and fibrinous necrotizing enterocolitis (Fig. 4). Mice infected by bacteria at the concentration of $1 \times 10^{6} \mathrm{CFU} / \mathrm{mouse} S$. typhimurium CVCC541 demonstrated similar phenomena, but the pathological changes were not severe (Fig. 4). In the mice infected by bacteria at the concentration of $1 \times 10^{5} \mathrm{CFU} /$ mouse and $1 \times 10^{4} \mathrm{CFU} / \mathrm{mouse}$, fewer absence of villi and inflammatory cells infiltration were observed, which indicated that the effect of bacteria with lower concentration on mice jejunum was mild (Fig. 4). The degree of pathological changes in the ileum (Fig. 5), colon (Fig. 6) and rectum (Fig. 7) was correlated with the infection dosage of bacteria. Mice in the negative control group did not have any pathological changes in the digestive tract. 


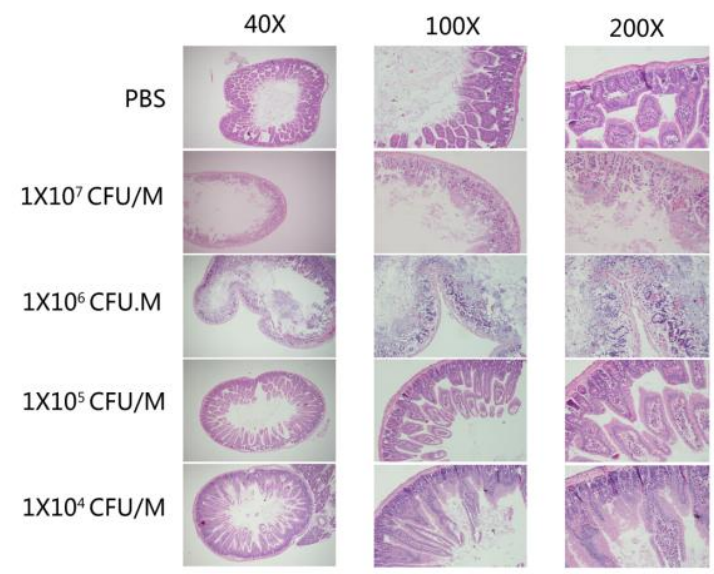

Fig. 4: The representative images of $\mathrm{H}-\mathrm{E}$ staining showing the pathological changes of the jejunum in mice infected with $\mathrm{S}$. typhimurium CVCC541.

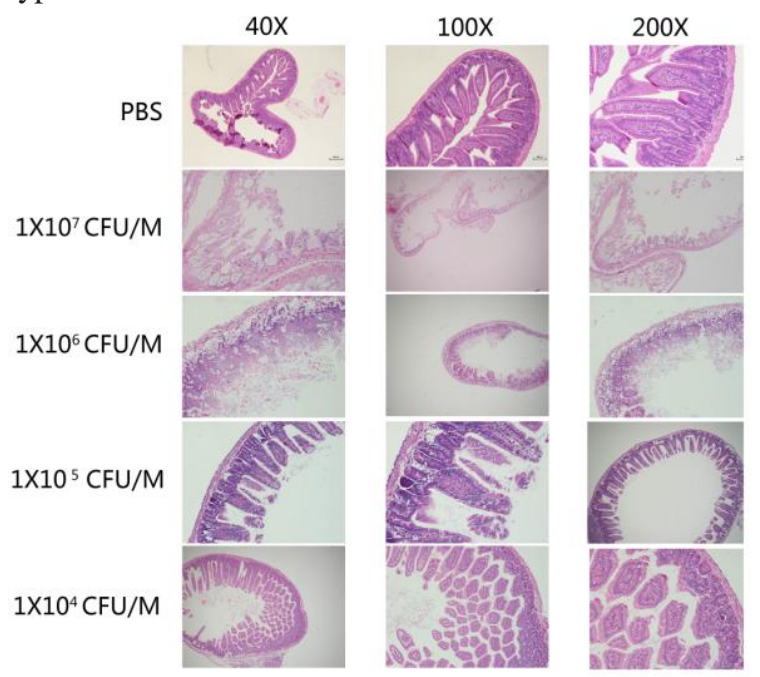

Fig. 5: The representative images of $\mathrm{H}-\mathrm{E}$ staining showing the pathological changes of ileum in mice infected with CVCC541.

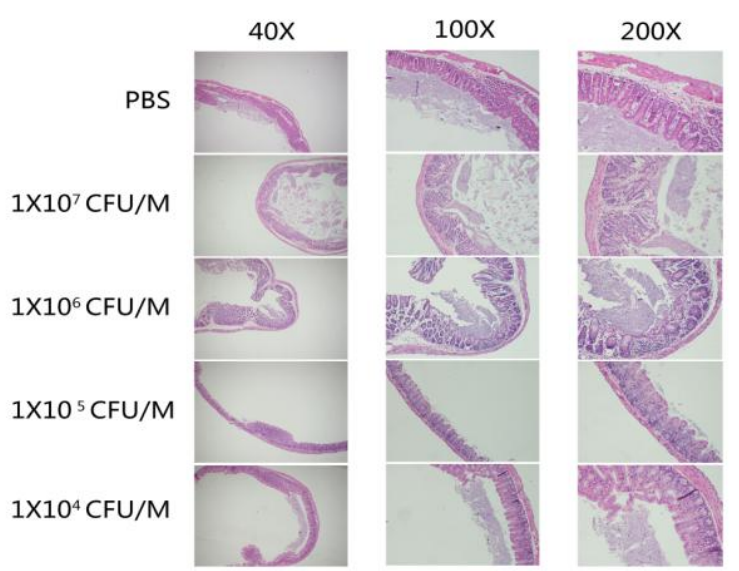

Fig. 6: The representative images of $\mathrm{H}-\mathrm{E}$ staining showing the pathological changes of colon in mice infected with CVCC541.

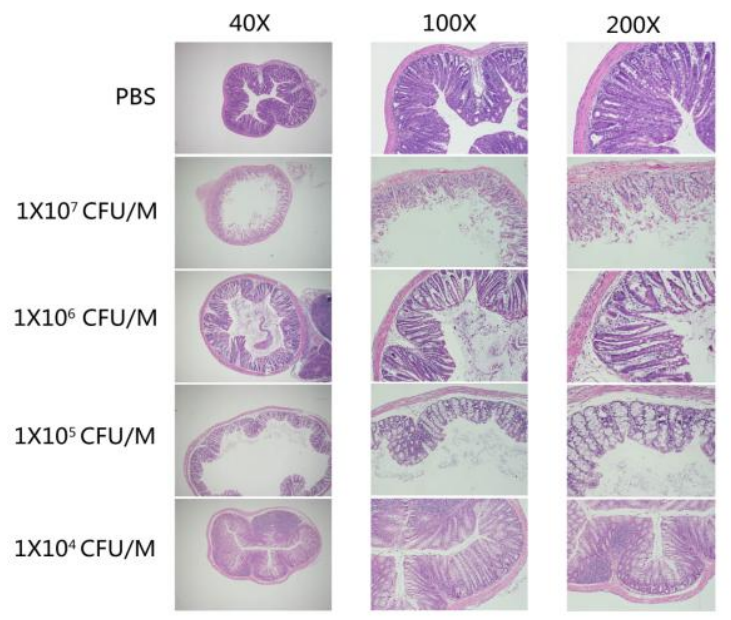

Fig. 7: The representative images of H-E staining showing the pathological changes of rectum in mice infected with CVCC541.

D. High concentration of S. typhimurium CVCC541 causes interstitial pneumonia in mice

The lung of mice infected by S. typhimurium CVCC541 was damaged to various degrees, and the injury was positively correlated with the dosage of $S$. typhimurium CVCC541. Acute interstitial pneumonia appeared in mice infected with S. typhimurium CVCC541 at the concentration of $1 \times 10^{7}$ CFU/mouse. The alveolar septa of mice became thickened because of congestion, edema and the infiltration of inflammatory cells. Alveoli collapsed, shrank, and disappeared. The neutrophils infiltrated (Fig. 8). The pathological changes of interstitial pneumonia also appeared in mice infected with $S$. typhimurium CVCC541 at the concentration of $1 \times 10^{6} \mathrm{CFU} / \mathrm{mouse}$ (Fig. 8). The pathological changes in mice infected with CVCC541 at the concentration of $1 \times 10^{5} \mathrm{CFU} /$ mouse and $1 \times 10^{4} \mathrm{CFU} /$ mouse were mild. Less local congestion in lung and less infiltration of red cells and inflammatory cells were noticed (Fig. 8). The pulmonary structure of mice in the negative control group was normal (Fig. 8).

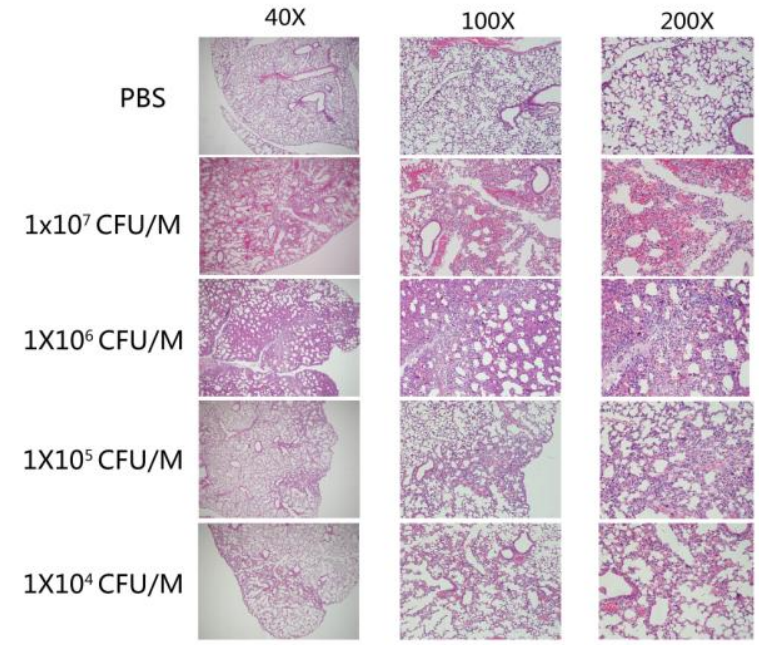

Fig.8 Representative images of H-E staining showing the pathological changes of the lung in mice infected with $S$. typhimurium CVCC541.

E. The dose dependent pathological changes in the spleen and liver in mice infected with S. typhimurium CVCC541 
In the spleen of mice infected with S. typhimurium CVCC541 at the concentration of $1 \times 10^{7} \mathrm{CFU} /$ mouse and $1 \times 10^{6}$ $\mathrm{CFU} /$ mouse, the symptoms of hemorrhagic splenomegaly were observed. Pathological changes included congestion, local necrosis, small splenic corpuscle, and infiltration of lots of neutrophils in splenic sinus. There was no significant pathological change in the spleen of mice infected with $S$. typhimurium CVCC541 at the concentration of $1 \times 10^{5}$ CFU/mouse and $1 \times 10^{4} \mathrm{CFU} / \mathrm{mouse}$ (Fig. 9). Severe necrotic foci, the degeneration and necrosis of hepatocytes with acute necrotic foci, hepatic cell disintegration, congestion, liver stasis, hepatic sinusoid extension and infiltration of red cells and neutrophils were observed in the liver of mice infected with S. typhimurium CVCC541 at the concentration of $1 \times 10^{7}$ $\mathrm{CFU} /$ mouse and $1 \times 10^{6} \mathrm{CFU} / \mathrm{mouse}$. The pathological changes in the liver of mice infected with $S$. typhimurium CVCC541 at the concentration of $1 \times 10^{5} \mathrm{CFU} /$ mouse and $1 \times 10^{4} \mathrm{CFU} /$ mouse were mild, and only scattered necrotic foci and infiltration of fewer amounts of red cells and neutrophils were observed. The liver structure of mice in the negative control groups was normal (Fig. 10).

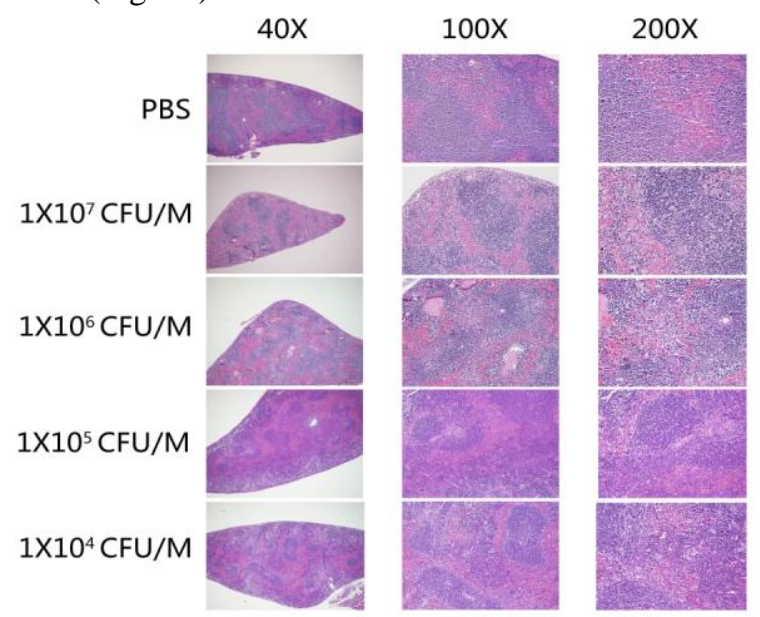

Fig. 9 Representative images of H-E staining showing the pathological changes of the spleen in mice infected with $\mathrm{S}$. typhimurium CVCC541.

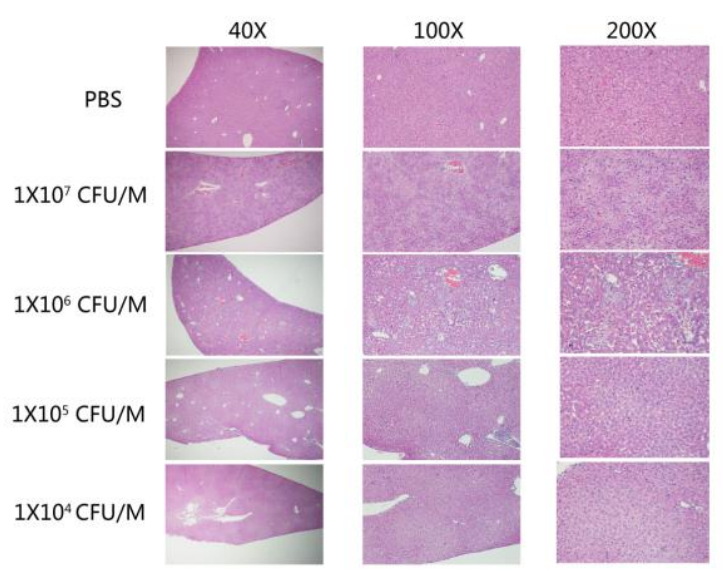

Fig. 10 Representative images of H-E staining showing the pathological changes of the liver in mice infected with $\mathrm{S}$. typhimurium CVCC541.

F. S. typhimurium CVCC541 could change the ratio of immune cells in the spleen
The ratios of $\mathrm{T}$ cells, neutrophils and macrophages were examined with flow cytometer. The results showed that the infection of S. typhimurium CVCC541 could significantly increase the ratio of neutrophils in the spleen of mice compared with control group $(51.3 \%$ vs $7.5 \%)$. This result was consistent with the histological pathological data, which indicated that the infection of $S$. typhimurium CVCC541 in mice could cause the inflammatory response in spleen. However, the infection of $S$. typhimurium CVCC541 decreased the ratios of macrophages and $\mathrm{T}$ cells in the spleen of mice to various degrees. Especially for T cells, the ratio of $\mathrm{T}$ cells in spleen decreased by two folds compared with control group $(18.0 \%$ vs $42.3 \%)$. With the progress of infection, the ratio of macrophage decreased from $34.8 \%$ to $22.5 \%$ (Fig. 11), which could be explained by the apoptosis of macrophages induced by $S$. typhimurium CVCC541 [19].
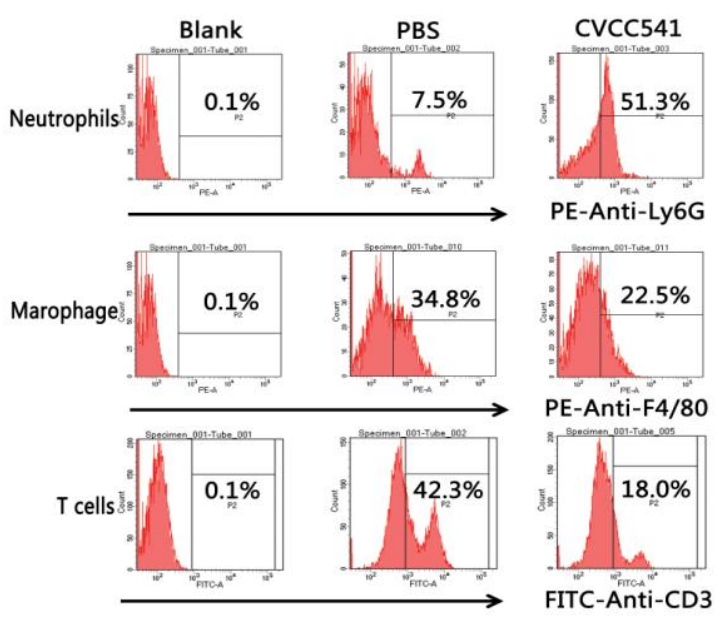

Fig. 11 The ratio changes of spleen immune cells in mice infected with S. typhimurium CVCC541 by flow cytometry.

\section{DISCUSSION}

S. typhimurium is an important anthropozoonosis, the epidemic and infection of $S$. typhimurium cause huge losses to the world economic development, and attract extensive social concerns [7], [20]. In our study, we established the mice infection model through infecting BALB/c mice with $S$. typhimurium CVCC541. The clinical symptoms of mice, pathological changes of organs, bacterial colonization in organs and the ratio of spleen lymphocytes were evaluated in detail. We found that the most infected organ was the intestine, especially centered in the small intestine area. The spleen and liver were rarely affected by $S$. typhimurium CVCC541 infection. Furthermore, our results thoroughly evaluated the severity of damage in mice caused by various infection dosages, which could provide the reference model for the study of the pathogenesis and molecular mechanism related to the drug resistance of Salmonella.

S. typhimurium is a type of facultative intracellular parasitic bacterium, which is very offensive to the gastrointestinal tract [21]. It has been reported that $S$. typhimurium mainly attacks the intestine after it infects mice, which could induce the dysfunction of gastrointestinal tract and the damage of gastrointestinal mucous membrane, were consistented with our results [22], [23]. After infected with S. typhimurium, 
most of mice have a systemic inflammatory response and an enlarged spleen [24]. Since S. typhimurium is a type of facultative intracellular pathogen, it could live in the macrophage [25], induce the apoptosis of macrophage, and release of mature proinflammatory cytokines, which could lead to the decrease of the macrophage ratio [26]. Moreover, $S$. typhimurium could produce L-asparaginase. L-asparaginase could deplete the amount of L-asparagine, which is an important nutrient for the activation of $\mathrm{T}$ cells. Therefore, S. typhimurium could inhibit the activation of T cells, and further decrease the ratio of $T$ cells [27]. Neutrophils in mice spleen increased after mice were chronically infected by $S$. typhimurium [28]. Neutrophils could kill the bacteria in both oxidative and non-oxidative manner [29]. therefore, S. typhimurium could be eliminated at higher efficacy by the increased ratio of neutrophils.

BALB/c mouse model infected by $S$. typhimurium could be used for developing new drugs. Based on the mouse model, the effect of drug composed of human defensins phBD-1 and phBD- 2 at a ratio of 2 to 1 was examined. The results indicated that this drug could decrease the colonization of $S$. typhimurium in the liver and spleen, and prolong the survival time of mice, which could be a potential candidate drug for the treatment of S. typhimurium (MTCC No. SL1344) [30]. The natural extract from Pomegranate Peel have been proven to cure the mice infected by S. typhimurium (ATCC 19943), and increase the survival rate of mice [31]. Proline peptide Bac7 could prolong the survival duration of mice infected by $S$. typhimurium (ATCC14028) at the concentration of $30 \mathrm{mg} / \mathrm{kg}$ [32]. In our study, for the first time, we successfully established the mouse model infected by $S$. typhimurium CVCC541, assessed the correlation between the symptoms of mice and the attacking concentration of bacteria in detail, and laid the foundation for the future study of drug resistance and pathogenic mechanism of $S$. Typhimurium.

\section{ACKNOWLEDGEMENT}

This work was supported by the National Key Research and Development Program of China(2016YFD0500708), the National Natural Science Foundation of China (No. 31672559).We thank Professor Hanna Fotina for her cooperation and support to the actualization of this work.

\section{REFERENCES}

[1] H. Herikstad, Y. Motarjemi, R. V. Tauxe. Salmonella surveillance: a global survey of public health serotyping. Epidemiol Infect, 129(1) (2002): 1-8.

[2] J. Lalsiamthara, J. H. Lee. Pathogenic traits of Salmonella Montevideo in experimental infections in vivo and in vitro. Scientific Reports, 7(46232) (2017): 46232.

[3] X. Y. Liu, R. Lu, Y. L. Xia, J. Sun. Global analysis of the eukaryotic pathways and networks regulated by Salmonella typhimurium in mouse intestinal infection in vivo. BMC Genomics, 11(1) (2010): 722.

[4] D. S. Chandel, R. Chaudhry, B. Dhawan, A. Pandey, A. B. Dey. Drug-resistant Salmonella enterica serotype Paratyphi A in India. Emerging infectious diseases, 6(4) (2000): 420-421.

[5] S. M. Pires, A. R. Vieira, T. Hald, D. Cole. Source Attribution of Human Salmonellosis: An Overview of Methods and Estimates. Foodborne Pathogens and Disease, 11(9) (2014): 667-676.

[6] D. Xiong, L. Song, J. Tao, H. J. Zheng, Z. Zhou, S. Z. Geng et al. An Efficient Multiplex PCR-Based Assay as a Novel Tool for Accurate Inter-Serovar Discrimination of Salmonella Enteritidis, S.
Pullorum/Gallinarum and S. Dublin. Frontiers in Microbiology, 8(420) (2017):

[7] H. J. Dong, S. Cho, D. Boxrud, S. Rankin, F. Downe, J. Lovchik et al Single-nucleotide polymorphism typing analysis for molecular subtyping of Salmonella Tennessee isolates associated with the 2007 nationwide peanut butter outbreak in the United States. Gut Pathogens, 9(1) (2017): 25

[8] D. Bumann. Pathogen proteomes during infection: A basis for infection research and novel control strategies. Journal of Proteomics, 73(11) (2010): 2267-2276.

[9] P. Broz, M. B. Ohlson, D. M. Monack. Innate immune response to Salmonella typhimurium, a model enteric pathogen. Gut microbes, 3(2) (2012): 62-70

[10] G. Sebastiani, V. Blais, V. Sancho, S. N. Vogel, M. M. Stevenson, P. Gros et al. Host immune response to Salmonella enterica serovar Typhimurium infection in mice derived from wild strains. Infection and immunity, 70(4) (2002): 1997-2009.

[11] P. Kalia, N. R. Kumar, K. Harjai. Studies on the therapeutic effect of propolis along with standard antibacterial drug in Salmonella enterica serovar Typhimurium infected BALB/c mice. BMC COMPLEMENTARY AND ALTERNATIVE MEDICINE, 16(485) (2016):

[12] L. Wang, W. H. Qin, S. X. Yang, R. D. Zhai, L. Zhou, C. J. Sun et al. The Adh adhesin domain is required for trimeric autotransporter Apa1-mediated Actinobacillus pleuropneumoniae adhesion, autoaggregation, biofilm formation and pathogenicity. Veterinary Microbiology, 177(1-2) (2015): 175-183.

[13] Y. Matsumoto, S. Miwa, Y. Zhang, M. Zhao, S. Yano, F. Uehara et al. Intraperitoneal administration of tumor-targeting Salmonella typhimurium A1-R inhibits disseminated human ovarian cancer and extends survival in nude mice. ONCOTARGET, 6(13) (2015): 11369-11377.

[14] H. Ozkaya, A. B. Akcan, G. Aydemir, S. Aydinoz, Y. Razia, S. T. Gammon, J. McKinney. Salmonella typhimurium infections in balb/c mice: a comparison of tissue bioluminescence, tissue cultures and mice clinical scores. NEW MICROBIOLOGICA, 35(1) (2012): 53-59.

[15] Q. He, J. K. Li, F. Li, R. G. Li, G. Q. Zhan, G. Li et al. Mechanism of action of gypenosides on type 2 diabetes and non-alcoholic fatty liver disease in rats. WORLD JOURNAL OF GASTROENTEROLOGY, 21(7) (2015): 2058-2066.

[16] L. Wang, W. H. Qin, J. Zhang, C. T. Bao, H. Zhang, Y. Y. Che et al. Adh enhances Actinobacillus pleuropneumoniae pathogenicity by binding to OR5M11 and activating p38 which induces apoptosis of PAMs and IL-8 release. SCIENTIFIC REPORTS, 6(24058) (2016):

[17] M. Bogunovic, F. Ginhoux, J. Helft, L. Shang, D. Hashimoto, M Greter et al. Origin of the Lamina Propria Dendritic Cell Network. IMMUNITY, 31(3) (2009): 513-525.

[18] D. J. I. Klinke, K. M. Brundage. Scalable Analysis of Flow Cytometry Data Using R/Bioconductor. CYTOMETRY PART A, 75A(8) (2009): 699-706.

[19] D. M. Monack, D. Hersh, N. Ghori, D. Bouley, A. Zychlinsky, S. Falkow. Salmonella exploits caspase-1 to colonize Peyer's patches in a murine typhoid model. The Journal of experimental medicine, 192(2) (2000): 249-258.

[20] X. Y. Liu, R. Lu, Y. L. Xia, J. Sun. Global analysis of the eukaryotic pathways and networks regulated by Salmonella typhimurium in mouse intestinal infection in vivo. BMC GENOMICS, 11(722) (2010):

[21] K. G. Watson, D. W. Holden. Dynamics of growth and dissemination of Salmonella in vivo. CELLULAR MICROBIOLOGY, 12(10) (2010): 1389-1397.

[22] M. Raffatellu, M. D. George, Y. Akiyama, M. J. Hornsby, S. Nuccio, T. A. Paixao et al. Lipocalin-2 Resistance Confers an Advantage to Salmonella enterica Serotype Typhimurium for Growth and Survival in the Inflamed Intestine. Cell Host \& Microbe, 5(5) (2009): 476-486.

[23] S. A. Seibert, P. Mex, A. Koehler, S. H. E. Kaufmann, H. Mittruecker. TLR2-, TLR4-and Myd88-independent acquired humoral and cellular immunity against Salmonella enterica serovar Typhimurium. IMMUNOLOGY LETTERS, 127(2) (2010): 126-134.

[24] K. L. Rosche, A. T. Aljasham, J. N. Kipfer, B. T. Piatkowski, V. Konjufca. Infection with Salmonella enterica Serovar Typhimurium Leads to Increased Proportions of F4/80+ Red Pulp Macrophages and Decreased Proportions of B and T Lymphocytes in the Spleen. PLOS ONE, 10(6) (2015): e130092.

[25] A. Richter-Dahlfors, A. M. Buchan, B. B. Finlay. Murine salmonellosis studied by confocal microscopy: Salmonella typhimurium resides intracellularly inside macrophages and exerts a cytotoxic effect on phagocytes in vivo. The Journal of experimental medicine, 186(4) (1997): 569-580 
[26] D. M. Monack, D. Hersh, N. Ghori, D. Bouley, A. Zychlinsky. Salmonella exploits caspase-1 to colonize Peyer\&\#39;s patches in a murine typhoid model. The Journal of experimental medicine, 192(2) (2000): 249-258.

[27] A. Torres, J. D. Luke, A. L. Kullas, K. Kapilashrami, Y. Botbol, A. Koller et al. Asparagine deprivation mediated by Salmonella asparaginase causes suppression of activation-induced T cell metabolic reprogramming. Journal of Leukocyte Biology, 99(2) (2016): 387-398.

[28] C. Johansson, M. Ingman, M. Jo Wick. Elevated neutrophil, macrophage and dendritic cell numbers characterize immune cell populations in mice chronically infected with Salmonella. Microbial Pathogenesis, 41(2-3) (2006): 49-58.

[29] B. R. Tuinema, S. A. Reid-Yu, B. K. Coombes. Salmonella Evades D-Amino Acid Oxidase To Promote Infection in Neutrophils. MBIO, 5(e018866) (2014):

[30] S. Patro, S. Maiti, S. K. Panda, N. Dey. Utilization of plant-derived recombinant human $\beta$-defensins (hBD-1 and hBD-2) for averting salmonellosis. Transgenic Research, 24(2) (2015): 353-364.

[31] J. Choi, O. Kang, Y. Lee, H. Chae, Y. Oh, O. Brice et al. In Vitro and In Vivo Antibacterial Activity of Punica granatum Peel Ethanol Extract against Salmonella. EVIDENCE-BASED COMPLEMENTARY AND ALTERNATIVE MEDICINE, (2011): 1-8.

[32] M. Benincasa, C. Pelillo, S. Zorzet, C. Garrovo, S. Biffi, R. Gennaro, M. Scocchi. The proline-rich peptide Bac7(1-35) reduces mortality from Salmonella typhimurium in a mouse model of infection. BMC Microbiology, 10(1) (2010): 178. 